\title{
The Impact of Physical Activity on Pericarditis
}

\author{
Jelani K. Grant ${ }^{1}$ - Nishant P. Shah ${ }^{2}$ \\ Accepted: 6 May 2021 / Published online: 27 August 2021 \\ (C) The Author(s), under exclusive licence to Springer Science+Business Media, LLC, part of Springer Nature 2021
}

\begin{abstract}
Purpose of Review To discuss the possible harmful effects and pathophysiology of exercise in cases of pericarditis, explore the role of multi-modality imaging to help guide exercise recommendations, and compare U.S. with European guideline recommendations on the safe resumption of physical activity following resolution of pericarditis.

Recent Findings Despite multiple postulated mechanisms by which exercise may be harmful during active inflammation of the myocardium or pericardium, the exact pathophysiology remains largely unknown. The inclusion of multi-modality cardiac imaging may play a role in further elucidating the relationship of exercise with outcomes in pericarditis. More recently, the prevalence of COVID-19 cardiac involvement in athletes prior to returning to play appears lower than previously reported; however, current recommendations are consistent with those for other etiologies of pericarditis.

Summary Further research is certainly needed to better understand the relationship between physical activity and pericarditis, the pathophysiology, and the prognostic role of multimodality imaging.
\end{abstract}

Keywords Pericarditis $\cdot$ Physical activity $\cdot$ Exercise $\cdot$ Return to play

\section{Introduction}

Acute pericarditis is the most common form of pericardial disease and refers to the inflammation of the pericardial sac [1]. Exact epidemiological data in the USA is limited; however, the incidence of acute pericarditis has been reported as 27.7 cases per 100,000 person-years in Northern Italy [2].

There are various etiologies of pericarditis largely grouped into idiopathic, infectious, and non-infectious causes [3]. In the developed world, the most common etiology is idiopathic or viral, whereas in the developing world, tuberculosis is the leading cause [4]. The diagnosis of pericarditis is based on a constellation of clinical symptoms, physical exam, EKG changes, laboratory abnormalities, and imaging findings. Treatment of acute pericarditis includes anti-inflammatory

This article is part of the Topical Collection on Pericardial Disease

Nishant P. Shah

nishant.shah@duke.edu

Jelani K. Grant

jelani.grant@jhsmiami.org

1 Department of Internal Medicine, University of Miami Miller School of Medicine/Jackson Memorial Hospital, Miami, FL, USA

2 Division of Cardiology, Duke Heart Center, Duke University, 2301 Erwin RD, Durham, NC 27705, USA agents, usually starting with non-steroidal anti-inflammatory drugs (NSAIDS) and colchicine, with steroids and immune modulators reserved for incessant, chronic, or recurrent cases [5-7]. However, there are also non-pharmacologic therapies, such as physical activity restriction that are thought to aid in healing. The rationale behind the avoidance of physical activity when diagnosed with pericarditis is to reduce the risk of complications such as progression to myocarditis, worsening pericardial effusion and cardiac tamponade, constrictive pericarditis, or recurrent/refractory symptoms. However, the literature is limited when it comes to understanding how exercise can further complicate cases of pericarditis and what level of physical activity is acceptable. In this review, we discuss the possible pathophysiology of exercise in acute pericarditis, explore the role of multi-modality imaging to help guide exercise recommendations, and compare U.S. with European guideline recommendations on the safe resumption of physical activity following resolution of acute pericarditis.

\section{The Pathophysiology and Impact of Physical Activity in Acute Pericarditis}

Current guidelines recommend non-competitive athletes should restrict physical activity until the resolution of symptoms and normalization of biomarkers while competitive 
athletes should not participate in competitive sports until resolution of symptoms and normalization of biomarkers. Specifically, this includes the absence of fever, absence of pericardial effusion, and normalization of erythrocyte sedimentation rate (ESR) and C-Reactive Protein (CRP) [8]. According to the 2015 European Society of Cardiology (ESC) Guidelines for the diagnosis and management of pericardial diseases and the 2019 position statement of the Sport Cardiology Section of the European Association of Preventive Cardiology (EAPC), athletes should be re-evaluated by a clinician prior to resuming training and competition $[9,10]$. However, these recommendations are largely based on expert consensus without trial evidence to support them. Some mechanisms behind the complications seen with physical activity in persons with active pericarditis seems to be immune mediated with some hypotheses being based on animal and autopsy studies that looked primarily at cases of myocarditis [11-14].

First, the adverse effects of exercise-induced tachycardia and shear stress on the pericardium may lead to worsening inflammation, or increased blood flow to the pericardium secondary to inflammation and resulting oxidative stress from free radicals (see Fig. 1) [15]. Studies have also reported a relationship between pericarditis and genetic variations of the immune system that can predispose people to worsening inflammation from environmental triggers such as exercise [15].

Next, although physical exercise is generally associated with improved health, there is a period after strenuous endurance activity that is associated with a functional immunodepression that can vary in length depending on the extent of the exercise [11]. Referred to as the 'open window' theory, this period could predispose individuals with pericarditis to other infectious agents or increase the severity of an active viral infection that progresses to metabolically active cells in the myocardium leading to a myocarditis, a condition known to increase the risk of sudden cardiac death $[12,16]$. Many components of the immune system exhibit change after prolonged heavy exertion. The increases in blood granulocyte and monocyte phagocytosis and interleukin-6 suggest a strong pro-inflammatory response, whereas the increases in cortisol and interleukin-1 receptor antagonist show that other antiinflammatory forces are also at work [17]. These data suggest that the immune system may be suppressed and stressed, albeit transiently, following prolonged endurance exercise (however still remains unproven in humans). If an infection or other source of inflammation is detected following the immunodepression period, an acute phase reaction can occur. This results in a surge of inflammation that mobilizes the humoral and cell mediated immune response causing cytolysis and necrosis $[13,14,18,19]$. The inflammatory response not only can delay healing but can progress symptoms or make them refractory.

Last, exercise is known to increase catabolic reactions within the body to break down protein, carbohydrates, and fatty acids as energy sources to maintain high performance. Unfortunately, inflammatory conditions like pericarditis also increase the body's demand for these energy sources [13]. Thus, continued exercise with pericarditis may accelerate muscle wasting and deconditioning. Therefore, in addition to a delay in healing, there may be a risk of worsening performance and predisposition to musculoskeletal injury $[13,20]$.

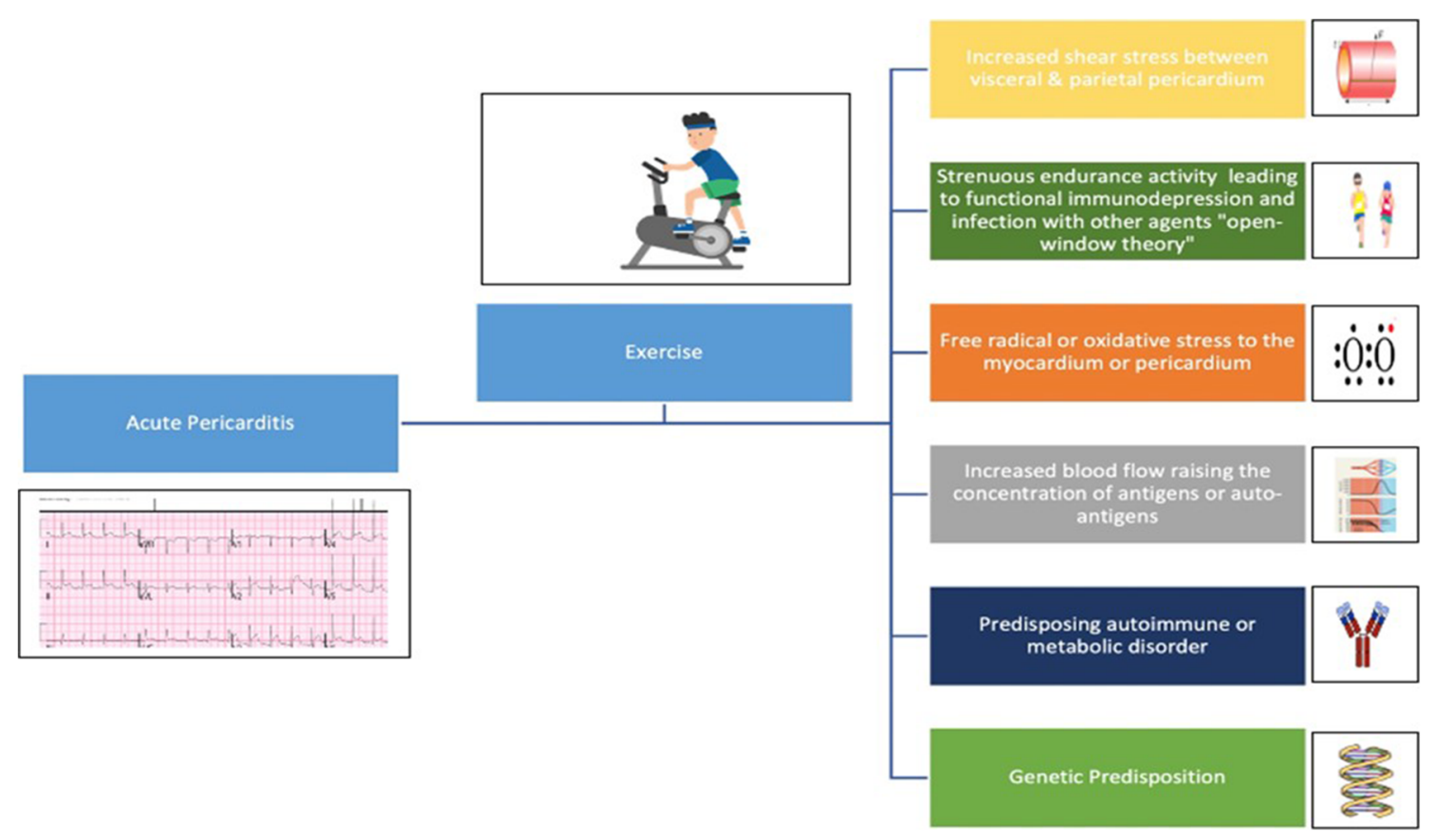

Fig. 1 Proposed mechanisms of the potential detrimental effect of exercise on the pericardium during active inflammation 
While these mechanisms have been hypothesized, it is important to remember that there are still no adequately controlled studies to further clarify exercise related injury in cases of pericarditis.

\section{The Role of Multi-Modality Imaging}

Individuals with pericarditis usually have an excellent prognosis with complete resolution of the pathological process; however, factors associated with a more guarded prognosis or increased risk of recurrence include a temperature $>100.4$ F on presentation, female sex, a subacute course, large pericardial effusion, resistance to NSAID use, and previous corticosteroid use $[10,21,22]$. Prior to 'return to exercise' recommendations for competitive athletes, a minimal restriction of 3 months has been defined, following which a normal workup to exclude active disease should be demonstrated. Multimodality imaging is an integral part of diagnosing and staging pericarditis as indicated by the European Association of Cardiovascular Imaging position statement and the American Society of Echocardiography Clinical Recommendations [23, 24]. More recently, there have been calls for including imaging in a concerted collaborative effort to understand more clearly the relationship of exercise with outcomes in pericarditis. Further studies in this space are certainly needed.

Transthoracic echocardiography is normal in $40 \%$ of acute pericarditis cases and can be useful in athletes with pericarditis to exclude complications such as pericardial effusions, in the setting of negative inflammatory biomarkers, to allow return to full activity [25]. Other findings may include increased pericardial brightness, pericardial thickening, and abnormal septal bounce, dilatation of the inferior vena cava and hepatic veins with absent or diminished inspiratory collapse, and pronounced respiratory variation in ventricular filling (mitral inflow velocity falls as much as $25 \%$ and tricuspid velocity greatly increases, $>40 \%$, in the first cardiac cycle following inspiration), suggesting constrictive pericarditis [23, 26]. More recent criteria have emphasized ventricular septal motion with respiration, mitral septal annular velocity $>8 \mathrm{~cm} / \mathrm{s}$, annular reversus, and hepatic vein end diastolic reversal flow velocity/forward diastolic velocity $>0.8$. to diagnose constriction [27].

On computed tomography (CT), noncalcified pericardial thickening with pericardial effusion is suggestive of acute pericarditis [23]. The pericardium can be visualized both in non-contrast and contrast-enhanced CTs, with enhancement of the thickened pericardium observed in cases of suspected pericarditis or tumor infiltration following administration of iodinated contrast media [24]. Secondary functional information, such as enlargement of the atria and vena cava in cases of pericardial constriction, may also be seen. CT attenuation values may help in the differentiation of exudative fluid (20 to 60 Hounsfield units), as found with purulent pericarditis, and simple transudative fluid ( $<10$ Hounsfield units) [24].

Late gadolinium enhancement (LGE) on cardiac magnetic resonance $(\mathrm{CMR})$ can also provide information on the presence and severity of active pericardial inflammation, with a sensitivity of nearly $94 \%$ (see Fig. 2) [29]. LGE is absent or minimal under physiological conditions because the normal pericardium is not vascularized, whereas acute pericarditis is associated with neovascularization [30]. LGE has been thought to improve, along with inflammatory biomarkers, with exercise restriction by most experts making CMR a useful imaging modality to prognosticate in cases of pericarditis, particularly those that are refractory or recurrent. CMR can also help evaluate pericardial thickness. In a recent multicenter observational cohort study of 128 consecutive patients with recurrent pericarditis evaluated by CMR during a 34-month mean follow-up, pericardial thickening (hazard ratio 2.6, 95\% confidence interval: 1.6 to 4.4 ) was more strongly associated with adverse outcomes compared to LGE (hazard ratio 0.3, $95 \%$ confidence interval 0.1 to 0.7 ) [31•]. While the authors did note a high specificity of LGE for active pericarditis and inflammation, its lower association with adverse events could be because the presence of LGE may have led to more targeted anti-inflammatory therapy leading to improved outcomes. In addition to evaluation for pericardial inflammation and thickening, CMR can also assess for signs of constrictive pericarditis, and its associated findings including calcification of the pericardium (though less reliably than CT), ventricular interdependence, and pericardial adhesions between the thickened pericardium and the epicardial surface of the myocardium with reduced mobility of the myocardium [24]. These findings may also be used to plan invasive treatment such as pericardiectomy.

Most patients with acute pericarditis will have a limited and uncomplicated illness, and echocardiography is the first and only imaging test necessary. However, after this initial risk stratification, a minority of patients will develop complicated pericarditis. Further imaging in cases of complicated pericarditis is directed at primarily answering two questions: First, does the patient still have significant pericardial inflammation? Second, does the patient have complications likes constriction or myocardial involvement? [32] Published reports are severely limited in this area; however, the data we do have suggest a helpful role of multimodality imaging and the utility of CMR in determining the stage and severity of pericardial inflammation. Thus, it is reasonable to consider additional imaging like CMR in all patients, not just athletes, with persistent symptoms to better understand prognosis of pericarditis. This may prove especially helpful in athletes when considering return to play. Transthoracic echocardiography and CMR together may also aid in the determination of whether myocardial involvement of pericarditis is present. Further 


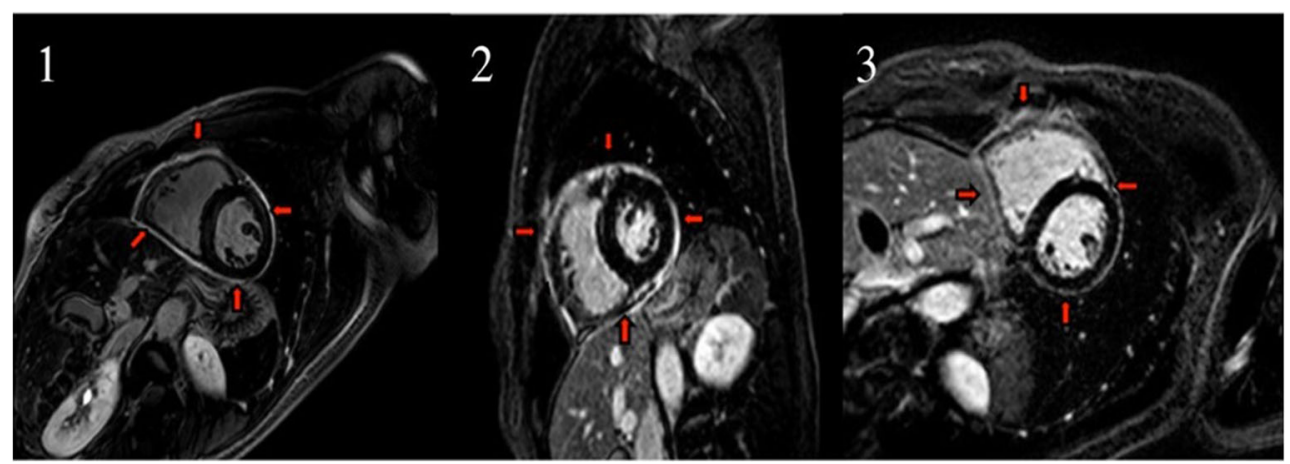

Fig. 2 (1) Initial cardiac magnetic resonance with late gadolinium enhancement (LGE) of the pericardium in a case of active pericarditis during anti-inflammatory therapy (red arrows); (2) cardiac magnetic resonance after ongoing exercise during medical therapy with worsening late gadolinium enhancement; (3) cardiac magnetic

research on subgroups that will benefit most from CMR for pericardial inflammation is certainly needed.

\section{Return to Play Specifically in COVID-19}

Some studies have suggested that acute pericarditis may also appear to be a common manifestation of SARS-coV-2 (COVID-19) cardiac involvement. One such study reported that $>33 \%$ of patients convalescing from COVID-19 had isolated pericardial enhancement either with or without underlying myocarditis, while a second study, though heavily criticized for the lack of a control CMR group, reported that $41 \%$ of athletes who had recovered from COVID-19 and underwent screening CMR imaging had evidence of pericardial hyperenhancement with associated pericardial effusions $[33,34]$. More recently, a large multicenter cross-sectional study $(n=798)$, between May and October 2020, sought to evaluate the prevalence of detectable inflammatory heart disease in professional athletes across major US professional sport leagues with prior COVID-19 infection, using current return to play screening recommendations [35••]. Abnormal screening results were identified in $30(3.8 \%)$ athletes (troponin, 6 athletes [0.8\%]; EKG, 10 athletes [1.3\%]; echocardiography, 20 athletes $[2.5 \%]$ ), necessitating additional testing. Of these requiring additional testing, 5 athletes $(0.6 \%)$ ultimately had CMR imaging findings suggesting inflammatory heart disease (myocarditis, 3; pericarditis, 2) which resulted in restriction from play.

This was a far lower prevalence of COVID-19 cardiac involvement in athletes prior to returning to play than previously reported [35••]. Perhaps, the pericardial involvement seen on CMR following COVID-19 infection may be directly related to the severity of infection. The study by Martinez et al. excluded patients with severe COVID-19 illness and reported findings for those who were asymptomatic or with mild symptoms. Alternatively, challenges may exist in CMR resonance after restriction of exercise without changes in medical therapy and improved late gadolinium enhancement. (Adapted from: Shah NP et al. JACC Cardiovasc Imaging 2019 Sep;12(9):1880-1881. doi: 10.1016/j.jcmg.2019.01.022. Epub 2019 Mar 13, with permission from Elsevier) [28]

interpretation, specifically differentiating attributes of athletic cardiac remodeling from potential cardiac pathology given the lack of athlete-specific CMR standards. Importantly, no adverse cardiac events were reported in athletes who underwent cardiac screening and resumed professional sport participation.

Despite these data, the pathophysiology and long-term effect of COVID-19-related pericarditis is unknown. Nevertheless, these findings suggest that CMR may be more of an adjunctive and downstream tool rather than frontline in determining return to play ability in athletes following COVID-19 infection. Current recommendations specific to exercise restriction with COVID-19-related pericarditis are consistent with the recommendations for other etiologies of pericarditis as discussed in this review [36]. The American College of Sports Medicine and the National Collegiate Athletic Association (NCAA) have adopted a gradual approach for athletes to return to activity (stage I: rest and recovery, stage II-IV: light, moderate, and intense activity, and stage V: normal training and full play); however, the timing of gradual advancement and strong evidence to support these recommendations are lacking [37]. It is important to ensure that there is no more evidence of active inflammation before increasing activity. More recently, "Long COVID" or "Longhauler COVID-19" are terms being used to describe illness in people who have either recovered from COVID-19 but are still report lasting effects of the infection or have had the usual symptoms for far longer than would be expected. The prevalence and impact of long standing COVID-19 symptoms on competitive athletes is also currently unknown.

\section{Comparison of U.S. and European Society Guideline Recommendations}

Current U.S. guidelines recommend exercise avoidance in individuals during active pericarditis with return to exercise 
after complete resolution of active disease. Whereas the European guidelines expanded on this recommendation stating: in competitive athletes return to exercise is only recommended after 30 days to 3 months, based on severity, with reevaluation before returning to sports [10, 38-40]. A comparison of the European Society of Cardiology (ESC) and American Heart Association (AHA) is shown in Table 1. Both guidelines recommend treating cases of acute pericarditis with myocardial involvement with the same recommendations for myocarditis. In the case of acute pericarditis with myocardial involvement, both the ESC and AHA suggest the same recommendations as those for myocarditis: abstinence from moderate- to high-intensity exercise for a period of 3-6 months, which may be guided by the presence of inflammation on T2-weighted images and LGE uptake on CMR. As per the 2015 AHA/ACC scientific statement on eligibility and disqualification recommendations for competitive athletes with cardiovascular abnormalities, chronic pericardial disease that results in constriction disqualifies the person from all competitive sports (Class III; Level of Evidence C) $[40]$.

\section{Future Directions}

Further research is certainly needed to better understand the relationship between physical activity and pericarditis, as well as the pathophysiology. More specifically, is the physical activity restriction meant for more moderate to vigorous exercise, or does it also include light activity? Furthermore, are certain subgroups more impacted than others, and can we leverage multimodality imaging to prognosticate and identify appropriate levels of activity? These questions are certainly very important to athletes who need to train and remain conditioned to be competitive. Despite the paucity of data, enough is known to encourage the avoidance of physical activity during active pericarditis with some experts recommending a heart rate of less than 100 beats per minute. Whether wearable devices should be used to monitor heart rate and stress load, or if resumption of activity should be slow or graded remains unknown, though a very important area to research. When counseling athletes on exercise restriction in cases of pericarditis, providers should be prepared to explain the possible mechanisms of injury associated with exercise in cases of pericarditis, complications that could occur, and recommendations from current guidelines. The use of multimodality imaging should also be considered in cases of persistent symptoms to help determine optimal timing of return to play.

Complications like recurrent pericarditis (RP) are associated with physical limitations and may occur following the first episode of acute pericarditis (affecting 15-30\% of patients). The chance of future recurrences increases with each additional recurrence [9]. The mainstay of current treatments includes NSAIDS, colchicine, and corticosteroids; however, newer

Table 1 Comparison of Current U.S. and European Return to Play Guidelines

2020 ESC Guidelines on Sports Cardiology and Exercise in Patients with 2015 ACC/AHA Scientific Statement on the Eligibility and

Cardiovascular Disease/ 2019 EAPC Sport Cardiology Section Position Disqualification Recommendations for Competitive Athletes with Statement

Cardiovascular Abnormalities

- Participation in leisure-time or competitive sports is not recommended for • Athletes with pericarditis, regardless of its pathogenesis, should not individuals with recent pericarditis while active inflammation is present (Class I, LOE C).

- Reassessment testing should include TTE to assess for pericardial effusions and measurement of inflammatory marker level.

- Return to all forms of exercise including competitive sports is recommended after 30 days to 3 months for individuals who have recovered completely ${ }^{\mathrm{a}}$, depending on clinical severity (Class III, LOE C).

- Athletes with concomitant myocardial involvement should be treated in accordance with the recommendations for myocarditis (Class IIa, LOE C). participate in competitive sports during the acute pericarditis phase (Class III, LOE C).

- Reassessment testing should include TTE to assess for left ventricular function, measurement of inflammatory markers, exercise EKG and 24-hour Holter monitoring.

- Athletes can return to full activity when there is complete absence of evidence for active disease, including effusion by echocardiography, and when serum markers of inflammation have normalized (Class III, LOE C).

- For pericarditis associated with evidence of myocardial involvement, eligibility should also be based on the course of myocarditis (Class III, LOE C).

- Asymptomatic athletes with small pericardial effusion, detected incidentally by imaging testing, but without evidence of myocardial inflammation, should not be considered as affected by myopericarditis and should not be restricted from sport participation. A periodical surveillance is however advisable (Class IIa, LOE C).

${ }^{a}$ Serum biomarkers have normalized, left ventricular function is normal, and there are no resting, or exercise-induced frequent/complex ventricular arrhythmias detectable on 24-h EKG monitoring or exercise EKG

$L O E$, level of evidence; TTE, transthoracic echocardiography; ESC, European Society of Cardiology; EAPC, European Association of Preventive Cardiology; ACC/AHA, American College of Cardiology/American Heart Association 
biologic agents such as rilonacept, which inhibits interleukin1 (IL-1) by binding to IL- $1 \alpha$ and IL- $1 \beta$, and other interleukin inhibitors may represent a paradigm shift in treatment, allowing a more targeted and personalized therapy for patients showing evidence of systemic inflammation. The RHAPSODY trial demonstrated the efficacy and safety of rilonacept in the management of symptomatic RP [28]. Perhaps biologics like these may provide a steroid sparing alternative and play a role in managing RP in several subgroups, including competitive athletes.

\section{Conclusion}

Despite multiple postulated mechanisms by which exercise may be harmful during active inflammation of the myocardium or pericardium, the exact pathophysiology remains largely unknown. The inclusion of multi-modality cardiac imaging may play a role in further elucidating the relationship of exercise with outcomes in pericarditis. At present, the ESC/EAPC and ACC/AHA guidelines recommend exercise avoidance in individuals during active pericarditis with return to exercise after complete resolution of active disease, and in the case of myocardial involvement, the same recommendations for myocarditis are encouraged.

\section{Declarations}

Conflict of Interest The authors confirm no relationship with industry, disclosures, or funding source for the work presented in this manuscript.

Human and Animal Rights and Informed Consent This article does not contain any studies with human or animal subjects performed by any of the authors.

\section{References}

Papers of particular interest, published recently, have been highlighted as:

- Of importance

•. Of major importance

1. Adler Y, Charron P, Imazio M, Badano L, Baron-Esquivias G, Bogaert J, et al. 2015 ESC Guidelines for the diagnosis and management of pericardial diseases. Task Force for the Diagnosis and Management of Pericardial Diseases of the European Society of Cardiology (ESC). G Ital Cardiol (Rome). 2015;16:702-38.

2. Imazio M, Cecchi E, Demichelis B, Chinaglia A, Ierna S, Demarie $\mathrm{D}$, et al. Myopericarditis versus viral or idiopathic acute pericarditis. Heart. 2008;94:498-501.

3. Imazio M, Demichelis B, Parrini I, Giuggia M, Cecchi E, Gaschino G, et al. Day-hospital treatment of acute pericarditis: a management program for outpatient therapy. J Am Coll Cardiol. 2004;43:1042-6.
4. Imazio M, Cecchi E, Demichelis B, Ierna S, Demarie D, Ghisio A, et al. Indicators of poor prognosis of acute pericarditis. Circulation. 2007;115:2739-44.

5. Imazio M, Brucato A, Trinchero R, Spodick D, Adler Y. Individualized therapy for pericarditis. Expert Rev Cardiovasc Ther. 2009; 7:965-75.

6. Imazio M, Brucato A, Trinchero R, Spodick D, Adler Y. Colchicine for pericarditis: hype or hope? Eur Heart J. 2009;30:532-9.

7. Imazio M, Brucato A, Belli R, Forno D, Ferro S, Trinchero R, et al. Colchicine for the prevention of pericarditis: what we know and what we do not know in 2014 - systematic review and metaanalysis. J Cardiovasc Med (Hagerstown). 2014;15:840-6.

8. Maron BJ, Zipes DP, Kovacs RJ. Eligibility and disqualification recommendations for competitive athletes with cardiovascular abnormalities: preamble, principles, and general considerations: a scientific statement from the American Heart Association and American College of Cardiology. J Am Coll Cardiol. 2015;66: 2343-9.

9. Adler Y, Charron P, Imazio M, Badano L, Barón-Esquivias G, Bogaert J, et al. 2015 ESC Guidelines for the diagnosis and management of pericardial diseases: the Task Force for the Diagnosis and Management of Pericardial Diseases of the European Society of Cardiology (ESC)Endorsed by: The European Association for Cardio-Thoracic Surgery (EACTS). Eur Heart J. 2015;36:2921-64.

10. Pelliccia A, Solberg EE, Papadakis M, Adami PE, Biffi A, Caselli $\mathrm{S}$, et al. Recommendations for participation in competitive and leisure time sport in athletes with cardiomyopathies, myocarditis, and pericarditis: position statement of the Sport Cardiology Section of the European Association of Preventive Cardiology (EAPC). Eur Heart J. 2019;40:19-33.

11. Mackinnon LT. Immunity in athletes. Int J Sports Med. 1997;18(Suppl 1):S62-8.

12. Fitzgerald L. Exercise and the immune system. Immunol Today. 1988;9:337-9.

13. Friman G, Wesslén L. Special feature for the Olympics: effects of exercise on the immune system: infections and exercise in highperformance athletes. Immunol Cell Biol. 2000;78:510-22.

14. McCaffrey FM, Braden DS, Strong WB. Sudden cardiac death in young athletes. A review. Am J Dis Child. 1991;145:177-83.

15. Lachmann HJ, Papa R, Gerhold K, Obici L, Touitou I, Cantarini L, et al. The phenotype of TNF receptor-associated autoinflammatory syndrome (TRAPS) at presentation: a series of 158 cases from the Eurofever/EUROTRAPS international registry. Ann Rheum Dis. 2014;73:2160-7.

16. Pedersen BK. Chronic exercise and the immune system. Exercise Immunology 1997:113-22.

17. Nieman DC. Influence of carbohydrate on the immune response to intensive, prolonged exercise. Exerc Immunol Rev. 1998;4:64-76.

18. Beisel KW, Srinivasappa J, Prabhakar BS. Molecular cloning of a heart antigen that cross-reacts with a neutralizing antibody to Coxsackievirus B4. Eur Heart J. 1991;12(Suppl D):60-4.

19. Huber SA, Gauntt CJ, Sakkinen P. Enteroviruses and myocarditis: viral pathogenesis through replication, cytokine induction, and immunopathogenicity. Adv Virus Res. 1998;51:35-80.

20. Maisch B. Exercise and sports in cardiac patients and athletes at risk: Balance between benefit and harm. Herz. 2015;40:395-401.

21. Imazio M, Bobbio M, Cecchi E, Demarie D, Demichelis B, Pomari $\mathrm{F}$, et al. Colchicine in addition to conventional therapy for acute pericarditis: results of the COlchicine for acute PEricarditis (COPE) trial. Circulation. 2005;112:2012-6.

22. Imazio M, Bobbio M, Cecchi E, Demarie D, Pomari F, Moratti M, et al. Colchicine as first-choice therapy for recurrent pericarditis: results of the CORE (COlchicine for REcurrent pericarditis) trial. Arch Intern Med. 2005;165:1987-91.

23. Klein AL, Abbara S, Agler DA, Appleton CP, Asher CR, Hoit B, et al. American Society of Echocardiography clinical 
recommendations for multimodality cardiovascular imaging of patients with pericardial disease: endorsed by the Society for Cardiovascular Magnetic Resonance and Society of Cardiovascular Computed Tomography. J Am Soc Echocardiogr. 2013;26:965-1012.e15.

24. Cosyns B, Plein S, Nihoyanopoulos P, Smiseth O, Achenbach S, Andrade MJ, et al. European Association of Cardiovascular Imaging (EACVI) position paper: multimodality imaging in pericardial disease. Eur Heart J Cardiovasc Imaging. 2015;16:12-31.

25. Maron BJ, Udelson JE, Bonow RO, Nishimura RA, Ackerman MJ, Estes NAM, et al. Eligibility and disqualification recommendations for competitive athletes with cardiovascular abnormalities: Task Force 3: hypertrophic cardiomyopathy, arrhythmogenic right ventricular cardiomyopathy and other cardiomyopathies, and myocarditis: a scientific statement from the American Heart Association and American College of Cardiology. J Am Coll Cardiol. 2015;66: 2362-71.

26. Sun JP, Abdalla IA, Yang XS, Rajagopalan N, Stewart WJ, Garcia $\mathrm{MJ}$, et al. Respiratory variation of mitral and pulmonary venous Doppler flow velocities in constrictive pericarditis before and after pericardiectomy. J Am Soc Echocardiogr. 2001;14:1119-26.

27. Welch TD, Ling LH, Espinosa RE, Anavekar NS, Wiste HJ, Lahr $\mathrm{BD}$, et al. Echocardiographic diagnosis of constrictive pericarditis: Mayo Clinic criteria. Circ Cardiovasc Imaging. 2014;7:526-34.

28. Klein AL, Imazio M, Cremer P, Brucato A, Abbate A, Fang F, et al. Phase 3 trial of interleukin-1 trap rilonacept in recurrent pericarditis. N Engl J Med. 2021;384:31-41.

29. Young PM, Glockner JF, Williamson EE, Morris MF, Araoz PA, Julsrud PR, et al. MR imaging findings in 76 consecutive surgically proven cases of pericardial disease with CT and pathologic correlation. Int J Card Imaging. 2012;28:1099-109.

30. Zurick AO, Bolen MA, Kwon DH, Tan CD, Popovic ZB, Rajeswaran J, et al. Pericardial delayed hyperenhancement with CMR imaging in patients with constrictive pericarditis undergoing surgical pericardiectomy: a case series with histopathological correlation. JACC Cardiovasc Imaging. 2011;4:1180-91.

31. Imazio M, Pivetta E, Palacio Restrepo S, Sormani P, Pedrotti P, Quarta G, et al. Usefulness of cardiac magnetic resonance for recurrent pericarditis. Am J Cardiol. 2020;125:146-51 Findings of this study suggest that LGE on CMR was associated with a lower incidence of adverse events. The authors postulated that this could be because the presence of LGE may have led to more targeted anti-inflammatory therapy leading to improved outcomes.

32. Cremer PC, Kumar A, Kontzias A, Tan CD, Rodriguez ER, Imazio $\mathrm{M}$, et al. Complicated pericarditis: understanding risk factors and pathophysiology to inform imaging and treatment. J Am Coll Cardiol. 2016;68:2311-28.

33. Puntmann VO, Carerj ML, Wieters I, Fahim M, Arendt C, Hoffmann J, et al. Outcomes of cardiovascular magnetic resonance imaging in patients recently recovered from Coronavirus Disease 2019 (COVID-19). JAMA Cardiol. 2020;5:1265-73.

34. Brito D, Meester S, Yanamala N, et al. High Prevalence of Pericardial Involvement in College Student Athletes Recovering From COVID-19. JACC Cardiovasc Imaging. 2021;14(3):54155. https://doi.org/10.1016/j.jcmg.2020.10.023

35. Martinez MW, Tucker AM, Bloom OJ, et al. Prevalence of Inflammatory Heart Disease Among Professional Athletes With Prior COVID-19 Infection Who Received Systematic Return-toPlay Cardiac Screening. JAMA Cardiol. 2021;6(7):745-52.

36. Phelan D, Kim JH, Elliott MD, Wasfy MM, Cremer P, Johri AM, et al. Screening of potential cardiac involvement in competitive athletes recovering from COVID-19: an expert consensus statement. JACC Cardiovasc Imaging. 2020;13:2635-52.

37. Stearns R S-MS, Huggins RA, et al. (June 2nd, 2020) Return to Sports and Exercise during the COVID-19 Pandemic: Guidance for High School and Collegiate Athletic Programs. Retrieved from: https://www.acsm.org/docs/default-source/covid-19-reopeningresources/return-tosports-and-exercise-during-covid-19-pandemic. pdf?sfvrsn $=12 \mathrm{~d} 644 \mathrm{db} 4$

38. Pelliccia A, Sharma S, Gati S, Bäck M, Börjesson M, Caselli S, et al. 2020 ESC Guidelines on sports cardiology and exercise in patients with cardiovascular disease. Eur Heart J. 2021;42:17-96.

39. Mitten MJ, Zipes DP, Maron BJ, Bryant WJ. Eligibility and disqualification recommendations for competitive athletes with cardiovascular abnormalities: Task Force 15: legal aspects of medical eligibility and disqualification recommendations: a scientific statement from the American Heart Association and American College of Cardiology. J Am Coll Cardiol. 2015;66:2447-50.

40. Maron BJ, Udelson JE, Bonow RO, Nishimura RA, Ackerman MJ, Estes NA, et al. Eligibility and disqualification recommendations for competitive athletes with cardiovascular abnormalities: Task Force 3: hypertrophic cardiomyopathy, arrhythmogenic right ventricular cardiomyopathy and other cardiomyopathies, and myocarditis: a scientific statement from the American Heart Association and American College of Cardiology. Circulation. 2015;132:e273-80.

Publisher's Note Springer Nature remains neutral with regard to jurisdictional claims in published maps and institutional affiliations. 